\title{
Metodologias Ativas integradas a um Sistema de Recomendação e Mineração de Dados Educacionais para a mitigação de evasão em EaD
}

\author{
Tiago Luís de Andrade ${ }^{1,2}$, Caroline Medeiros Martins de Almeida ${ }^{2}$, Jorge Luís Victória Barbosa ${ }^{2}$, \\ Sandro José Rigo ${ }^{2}$ \\ ${ }^{1}$ Universidade do Estado de Mato Grosso (UNEMAT) \\ ${ }^{2}$ Universidade do Vale do Rio dos Sinos (UNISINOS) \\ tiago@unemat.br, \{carolinemalmeida, jbarbosa, rigo\}@unisinos.br
}

\begin{abstract}
The high dropout rates are a concern for teachers and managers of Distance Education. There are initiatives to mitigate this situation, such as Educational Data Mining (EDM) and the use of Recommendation Systems (RS). Although effective in specific aspects, these techniques lack mechanisms to motivate students. Therefore, this article describes an SR model that presents as differential the use of Active Methodologies in order to mitigate the evasion risks of students identified through EDM techniques and enhance the permanence. A prototype was implemented and a functionality and acceptance evaluation was performed. According to the TAM model, more than $87 \%$ of the teachers agree with the ease of use and $77 \%$ agree that the $R S$ can be useful in the teaching and learning process of the students.
\end{abstract}

Resumo. Os altos índices de evasão preocupam docentes e gestores da Educação a Distância. Existem iniciativas para mitigação desta situação, como a Mineração de Dados Educacionais (MDE) e o uso de Sistemas de Recomendação (SR). Apesar de efetivas em aspectos especificos, estas técnicas carecem de mecanismos para motivação dos alunos. Diante disso, esse artigo descreve um modelo de SR que apresenta como diferencial o uso das Metodologias Ativas com o objetivo de mitigar os riscos de evasão dos alunos identificados através das técnicas de MDE e potencializar a permanência. Foi implementado um protótipo e realizada a avaliação de funcionalidade e aceitação. De acordo com o modelo TAM, mais de $87 \%$ dos docentes concordam com a facilidade de uso e 77\% concordam que o SR pode ser útil no processo de ensino e aprendizagem dos alunos.

\section{Introdução}

O elevado índice de evasão de estudantes nos cursos ofertados na modalidade de ensino a distância preocupa os gestores e professores das instituições de ensino, que buscam alternativas para identificar as situações passíveis de desistências e motivar os alunos a permanecerem nos estudos. Nesse contexto, existem trabalhos que utilizam as técnicas de Mineração de Dados Educacionais (MDE) para identificar alunos propensos a evadir [Marques et al. 2019], no entanto são restritas à identificação dessas possibilidades, sem posterior ação efetiva [Widyahastuti e Tjhin 2018]. Após o processo de identificação de uma tendência de evasão, a tomada de decisão para mitigar esse problema é normalmente dependente do professor ou gestor, que necessitam utilizar de metodologias educacionais para resgatar e incentivar o aluno a permanecer no curso.

A Metodologia Ativa é uma abordagem em que os alunos participam como protagonistas do processo de aprendizagem, estimulados ao relacionamento com os colegas para o desenvolvimento de atividades, colaborando para o crescimento intelectual e a melhoria no desempenho dos envolvidos [Guo et al. 2018]. Uma das hipóteses de prevenção de evasão na Educação a Distância $(\mathrm{EaD})$ é a utilização de Metodologias Ativas integradas a Sistemas de Recomendação (SR) e Ambientes Virtuais de Aprendizagem (AVA), de forma a auxiliar os professores no processo de ensino e aprendizagem, potencializando a permanência [Chandrasekaran et al. 2016] [Lima e Siebra 2017]. 
Estudos avaliam que a integração de Metodologias Ativas ao SR pode constituir um mecanismo efetivo na retenção, ampliando o potencial de engajamento dos alunos e de compartilhamento de aprendizagem [Lima e Siebra 2017], [Leite et al. 2019]. Uma vez identificados os casos associados com riscos de evasão por MDE, as Metodologias Ativas fomentam a colaboração dos estudantes por meio de diversos recursos, desde materiais recomendados para a leitura até a interação com o ambiente virtual, estimulando-os com relação ao seu aprendizado. Portanto, seria um recurso que, segundo Cunha e Siebra [2016] e Andrade et al. [2021], pode contribuir para a aprendizagem colaborativa e atuar na mitigação da evasão. Entretanto, conforme Leite et al. [2019], a utilização de Metodologias Ativas ainda é pouco observada na Educação a Distância.

Diante deste cenário, esse artigo apresenta uma proposta de modelo de SR que integra a abordagem pedagógica das Metodologias Ativas com o suporte das técnicas de MDE para mitigar os riscos de evasão e potencializar a permanência dos alunos. O principal diferencial desse trabalho consiste na integração de uma etapa motivacional ao trabalho dos alunos, utilizando-se para isso as Metodologias Ativas.

Este artigo está organizado em 5 seções. A seção 2 destaca trabalhos relacionados ao tema de pesquisa. A seção 3 propõe um modelo de SR integrado a Metodologias Ativas com suporte de MDE. A seção 4 apresenta o funcionamento, a avaliação de aceitação e a análise crítica da proposta. Por fim, a seção 5 contempla as considerações finais e sugestões de trabalhos futuros.

\section{Trabalhos Relacionados}

Essa seção apresenta trabalhos que abordam a utilização de Metodologias ativas, MDE e SR no contexto de cursos de Educação a Distância. Diante do estudo, verificou-se a possibilidade de expansão no uso de tecnologias e métodos nessa área.

Campos et al. [2017] citaram exemplos de SRs, como PMoodle, Broad-RSI, CA-Learning, LORSys, Dica, Mobile, e-Lors, RecoaComp, SR Colaborativa Móvel. Rolim et al. [2017] apresentaram um sistema capaz de classificar por meio de aprendizagem de máquina a postagem do aluno em fóruns do AVA e recomendar um material de estudo auxiliar disponibilizado como vídeos do Youtube. Acosta et al. [2018] desenvolveram um sistema que utiliza como método de ensino a Aprendizagem Baseada em Projetos com foco no aluno e colaboração entre pares, capaz de sugerir materiais complementares a partir de atividades propostas pelo professor nesse mesmo ambiente. Ferreira et al. [2015] propuseram um modelo de recomendação de conteúdo chamado UbiGroup para grupo de aprendizes com o objetivo de recomendar Objetos de Aprendizagem (OA) conforme os perfis e o contexto que estão inseridos, apoiando o professor na busca e seleção de materiais.

Em se tratando de MDE, abordagens de pesquisa como técnicas, algoritmos e atributos são os temas mais frequentes nos trabalhos estudados. As técnicas de classificação e predição são amplamente utilizadas para a análise do comportamento de aprendizagem e desempenho dos alunos, como apresentados nos trabalhos de Ramos et al. [2018], Kostopoulos et al. [2019] e Queiroga et al. [2019], possibilitando intervenções oportunas e eficazes na mitigação de evasão. Em relação a algoritmos, Random Forest e Logistic Regression são utilizados para a predição e detecção de riscos de evasão nos trabalhos de Ramos et al. [2017], Queiroga et al. [2019] e Waheed et al. [2020]. Por fim, sobre os atributos de pesquisa, Kostopoulos et al. [2019] investigaram os dados demográficos, que representam as características de perfil dos alunos; Santos et al. [2016], Ramos et al. [2018] e Brito et al. [2019] exploraram os dados de desempenhos, como notas obtidas em questionários e avaliações.

O uso de Metodologias Ativas está se expandindo no ensino presencial e a distância, pois apresenta um potencial de tornar as aulas mais interessantes e dinâmicas [Almeida et al. 2019] [Leite e Ramos 2017]. Lima et al. [2020] constataram que 76,7\% dos professores buscaram aprimorar seus métodos de ensino com a utilização de Métodos Ativos. Chandrasekaran et al. [2016] demonstraram que $67 \%$ dos entrevistados sentiram-se confortáveis em utilizar a metodologia no processo de aprendizagem, pois estimula a autonomia e interação, incentivando- 
os a aprender com o outro, a perceber e desenvolver a autoaprendizagem e a comunicação em grupos de trabalho. Quanto ao professor, os métodos ativos auxiliaram no processo de ensino e aprendizagem, tornando-o mais proativo e capaz de melhorar o desempenho dos alunos com ações que minimizam os riscos de desistência.

Não foram encontrados exemplos do uso de Metodologia Ativa integradas com técnicas de MDE. Consequentemente, nenhum dos trabalhos considerou integrar essas técnicas à Metodologias Ativas em um SR visando auxiliar o professor no ensino e apoiar a aprendizagem do aluno. Diante disso e com a finalidade de propor ações efetivas de mitigação da evasão, será descrito a seguir um modelo de SR que utiliza as Metodologias Ativas e técnicas de MDE. Busca-se avanços no processo de personalização do ensino e aprendizagem. Partindo da caracterização dos alunos de acordo com o seu histórico de atuação, o SR efetua um processo tradicional de recomendação, mas complementarmente instiga o uso de OA, a leitura de materiais adicionais, além da comunicação e interação entre os usuários no ambiente virtual. Inclui, portanto, elementos de impulso à autonomia, estimulando também práticas colaborativas.

\section{Modelo proposto}

O modelo proposto envolve um conjunto de etapas que são executadas de forma integrada e que se diferenciam nos aspectos de disponibilidade de recursos, dando o suporte aos alunos e professores. A seguir será apresentada a descrição geral do modelo e a sua implementação.

\subsection{Descrição geral do modelo}

A figura 1 ilustra o modelo de SR na visão do professor, que envolve o uso de Metodologias Ativas e técnicas de MDE.

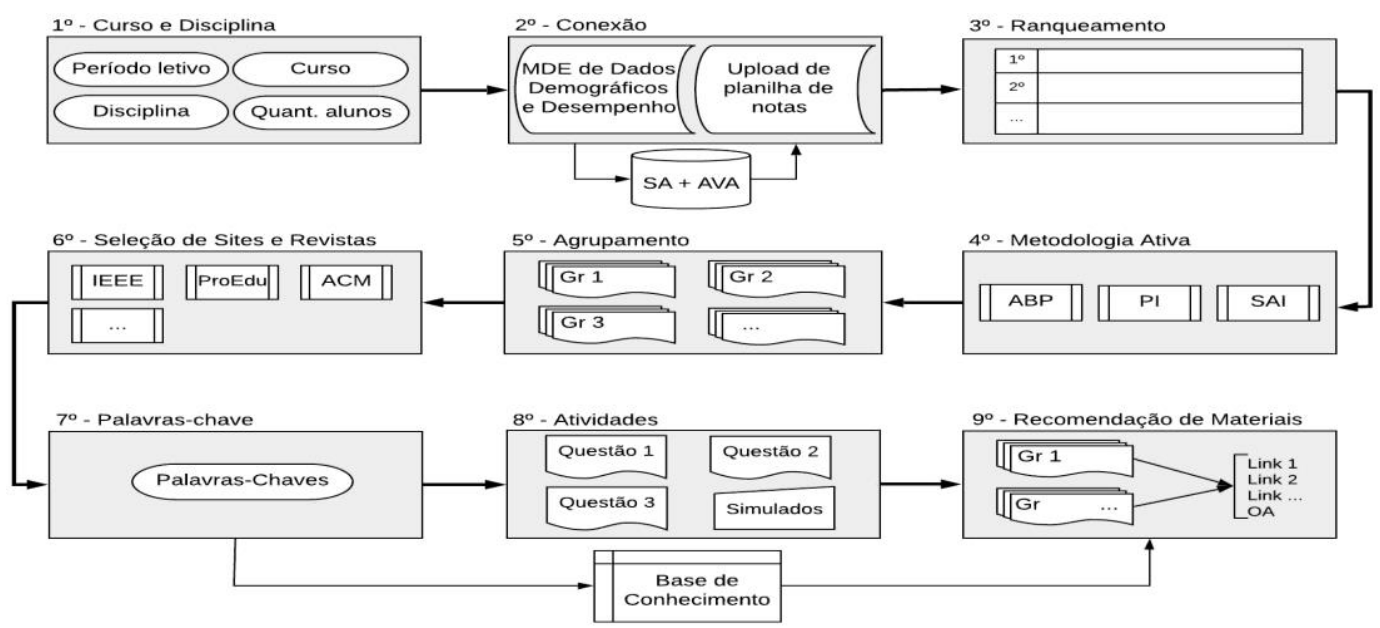

Figura 1. Modelo de SR com Metodologias Ativas e técnicas de MDE - visão do professor

O módulo Curso e Disciplina atua no cadastramento do nome do curso, disciplina, período letivo de oferta e quantidade de alunos matriculados. O módulo Conexão possui duas funcionalidades a serem escolhidas pelo professor. A primeira, MDE, integra a base de dados do AVA e Sistema Acadêmico (SA) para o acesso a dados demográficos, registros de perfil acadêmico e média do desempenho nas atividades dos alunos. Uma vez obtidos, utiliza-se a técnica de aprendizado supervisionado com os algoritmos Random Forest [Breiman 2001] e Naive Bayes [Lewis 1998] para gerar padrões de tendências a serem observadas. A segunda funcionalidade possibilita a utilização do quadro de notas extraído do AVA com os dados demográficos e desempenhos.

O módulo Ranqueamento envolve a classificação dos alunos em ordem decrescente conforme a média das atividades avaliativas. O módulo Metodologia Ativa permite ao professor a escolha da quantidade de alunos por grupo e a estratégia de aprendizagem, sendo que alguns dos exemplos possíveis são: (i) Aprendizagem Baseada em Problema - ABP [Silva e Silva 
2020], (ii) Sala de Aula Invertida - SAI [Bergmann e Sams 2016] ou (iii) Peer Instruction - PI [Araújo e Mazur 2013]. A escolha deve ser feita a cada realização das atividades avaliativas, uma vez que interfere diretamente na média da disciplina, e consequentemente, na formação dos grupos. Tal módulo visa auxiliar no desenvolvimento de diversas competências e habilidades, que no mundo contemporâneo cada vez é mais requisitado. O módulo Agrupamento possibilita ao professor obter apoio na formação de grupos de alunos, que segundo Pallof e Pratt [2002], quando os alunos trabalham colaborativamente, tendem a produzir um conhecimento mais profundo, pois deixam de ser independentes para se tornarem interdependentes.

O módulo Seleção de Sites e Revistas permite o professor escolher as revistas ou sites nacionais tais como Portal Domínio Público ${ }^{1}$, Banco Internacional de Objetos Educacionais ${ }^{2}$, ProEdu $^{3}, \mathrm{EduCAPES}^{4}$, e as internacionais IEEE ${ }^{5}, \mathrm{ACM}^{6}, \mathrm{Scielo}^{7}, \mathrm{Merlot}^{8}$, Google Scholar ${ }^{9}$, para a busca de materiais complementares para a leitura e aperfeiçoamento, como artigos, vídeos e OA. O módulo Palavras-Chave consiste no cadastro de palavras-chave relacionadas ao conteúdo da disciplina, utilizadas para a busca de materiais complementares. O módulo Atividades propõe o cadastramento de questões de pesquisa ou problemas para a resolução dos grupos formados, com o suporte por meio da leitura dos materiais complementares e a realização das atividades simuladas cadastradas nessa etapa como atividades de reforço.

O módulo Recomendação de Materiais consiste na seleção dos materiais complementares e OAs retornados pelos algoritmos de busca implementados por meio da Biblioteca BeautifulSoap, conforme o cadastro de palavras-chave e indicação dos sites e revistas. $\mathrm{O}$ professor pode assinalar entre os resultados o material que será apresentado no SR a nível de visão do aluno, formando uma base de conhecimento para futuras sugestões. O SR permite a visualização e extração de relatórios que demostram a participação e execução das atividades por integrantes dos grupos através dos dados de $\log$ de acesso e verificação de preenchimento das atividades. Nesse trabalho utiliza-se o modelo de SR Híbrido. Os dados dos usuários usados nas recomendações são coletados de forma explícita ou implícita, de registros e interações.

A figura 2 apresenta os componentes disponíveis ao aluno, compondo um conjunto de módulos integrados que são exibidos em uma única interface.

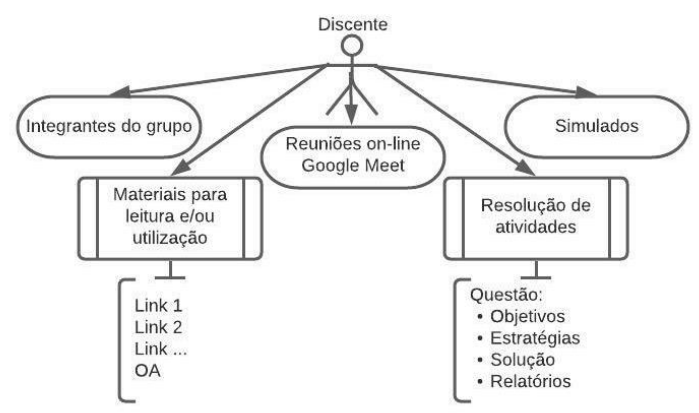

Figura 2: Modelo de integração do SR, a nível de visão do aluno

O módulo Integrantes do grupo exibe o nome e o e-mail de contato de todos os participantes do grupo, definidos conforme os critérios estabelecidos pelo professor e agrupamentos realizados pelo SR. O módulo Reuniões on-line permite a realização de webconferências a qualquer horário via plataforma de comunicação (por exemplo, Google Meet),

\footnotetext{
${ }^{1} \mathrm{http}: / /$ www.dominiopublico.gov.br/

${ }^{2} \mathrm{http}: / /$ objetoseducacionais.mec.gov.br/

${ }^{3} \mathrm{http}: / /$ proedu.rnp.br/

${ }^{4} \mathrm{https} / / /$ educapes.capes.gov.br/

${ }^{5}$ https://www.iee.org/index.html.

${ }^{6} \mathrm{http}: / / \mathrm{dl} . \mathrm{acm} . \mathrm{org} /$

${ }^{7}$ https://www.scielo.org/

${ }^{8} \mathrm{https}: / / \mathrm{www} . \mathrm{merlot}$.org/merlot/

${ }^{9} \mathrm{https} / / /$ scholar.google.com.br/
} 
que pode ser compartilhada pelos integrantes do grupo e demais convidados, inclusive o professor da disciplina, de forma a acompanhar o desenvolvimento e incentivar a troca de experiências, aprendizagens e discussão de dúvidas.

O módulo Simulados permite o acesso as atividades cadastradas pelo professor para o reforço do aprendizado. Para isso, os alunos também podem contar com o módulo Reuniões online, que permite web-conferências para a resolução e compartilhamento de conhecimentos. $\mathrm{O}$ módulo Materiais para a leitura elou utilização apresenta os links de artigos ou OA previamente selecionados pelo professor em sites e revistas nacionais e internacionais, que servem para o embasamento teórico/prático para a resolução das atividades.

O módulo Atividades para a realização apresenta questões ou problemas definidos pelo professor para que o grupo resolva. Exige-se o preenchimento dos objetivos, estratégias, solução e a postagem de um relatório descritivo das atividades realizadas. Ressalta-se que essas podem ser realizadas individualmente e a qualquer momento, no entanto, é importante destacar que os relatórios apresentam os registros de acessos via log que demonstram a participação e execução das atividades por integrantes do grupo.

\subsection{Implementação}

Baseado no modelo proposto foi desenvolvido um protótipo do SR seguindo o padrão arquitetônico Model-View-Controller $(M V C)$, com a linguagem de programação Python, os Frameworks Django e BootStrap, as Bibliotecas JQuery e BeautifulSoap, e, como banco de dados, SQLite. Os componentes do SR podem ser vistos na figura 3.

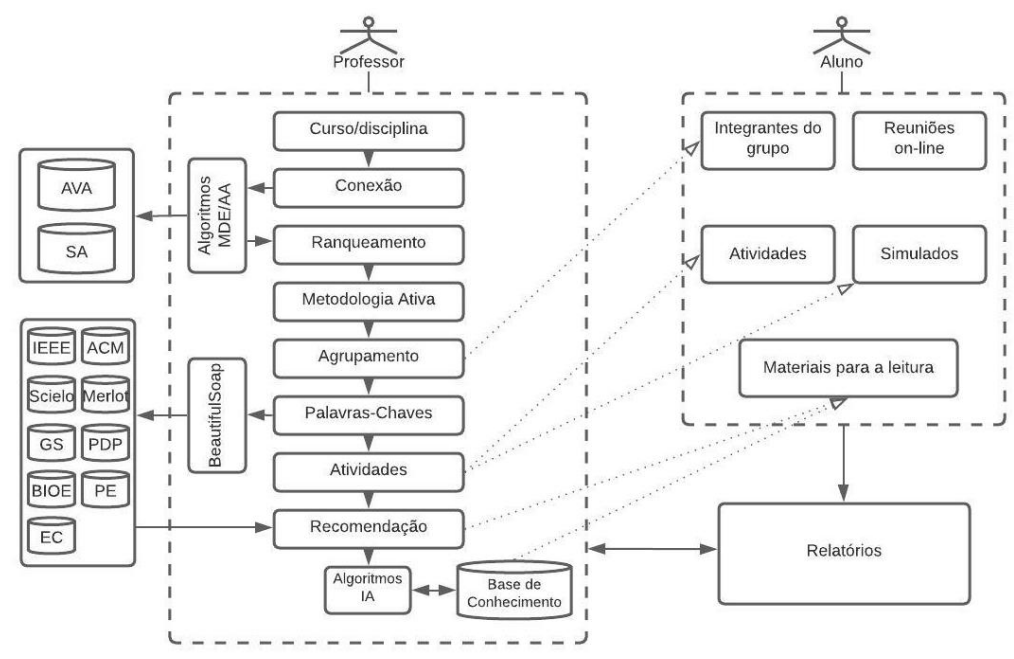

Figura 3: Componentes do SR

A interface do professor necessita conectar a base de dados do AVA e SA para a coleta de dados demográficos e de desempenho logo após a realização da primeira avaliação da disciplina ofertada. Nessa interface é necessário consultar as bases de dados dos sites e revistas eletrônicas nacionais e internacionais para a identificação de materiais complementares e objetos de aprendizagens que serão recomendados aos alunos. Já na interface do aluno os dados apresentados são resultados dos cadastros realizados pelo professor e as consultas as bases de dados externas dos sites e revistas eletrônicas.

Inicialmente uma interface comum exige o cadastro e autenticação, seguido do preenchimento de dados pessoais e identificação do perfil. Após o acesso, na interface do professor é possível cadastrar, visualizar e editar os dados da disciplina ofertada; na interface do aluno, visualizar as disciplinas vinculadas e conteúdos cadastrados pelo professor.

\section{Estudo de caso e avaliação}

O estudo de caso foi realizado com professores de duas universidades, localizadas no centro-oeste e no sul do Brasil. A primeira avaliação consistiu na verificação das 
funcionalidades e apresentação das interfaces, realizada por um professor que ministra a disciplina de Introdução a Banco de Dados a uma turma real de 30 alunos. Por sua vez, a segunda focou na aceitação através de um questionário conforme critérios do modelo TAM Technology Acceptance Model [Marangunié e Granié 2014].

\subsection{Avaliação de funcionalidade e interfaces do SR}

A primeira etapa de uso do SR consiste no cadastro de dados do curso e disciplina ofertada pelo professor. O período letivo definido foi 2021/1, curso de Ciência da Computação, disciplina de Introdução a Banco de Dados e a quantidade de 30 alunos. Na segunda etapa foi realizado o upload do quadro de notas do AVA, que contém os dados demográficos e de desempenho dos alunos matriculados. Posteriormente, na terceira etapa, os alunos foram classificados em ordem decrescente e exibidos em uma lista ao professor contendo nome, sobrenome, endereço de email e nota dos alunos antes e depois da classificação.

$\mathrm{Na}$ quarta etapa, no módulo Metodologia Ativa, a quantidade de 5 alunos por grupo foi escolhida pelo professor e o método Aprendizagem Baseada em Problema (ABP), conforme a figura 4, que segundo Alves et al. [2020], tende a privilegiar os conhecimentos prévios dos estudantes, incentivando um ambiente investigativo de aprendizagem através da construção de hipóteses e experimentações.

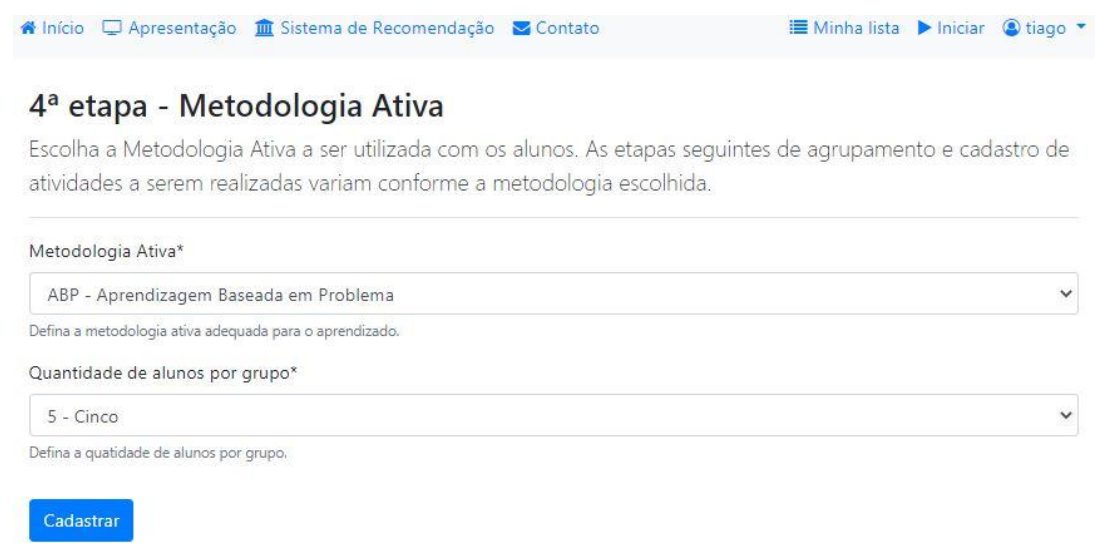

Figura 4: Interface do módulo Metodologia Ativa

A quinta etapa foi responsável pela formação de grupos, conforme definidos na etapa anterior pelo professor, com a apresentação dos integrantes e seus respectivos contatos, os quais terão atividades a serem realizadas e contarão com a indicação de materiais para a leitura e OA.

A sexta etapa, módulo Seleção de Sites e Revistas, o professor pode escolher uma ou mais bases de dados de sites e revistas para que o SR busque por materiais complementares. No caso, foi escolhida a base de dados da IEEE - Institute of Electrical and Electronics Engineers, conforme figura 5.

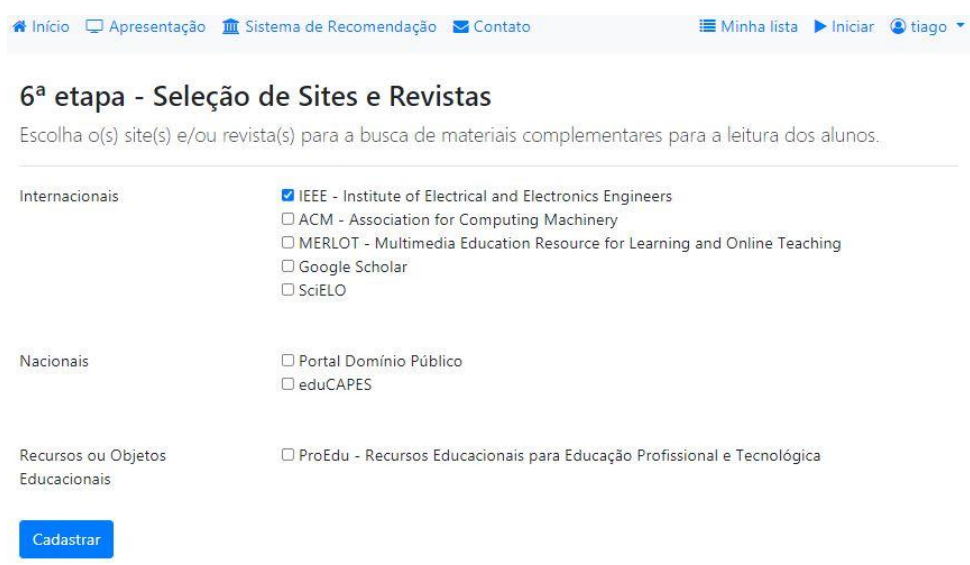

Figura 5: Interface do módulo Seleção de Sites e Revistas 
Na sétima etapa, Cadastro de Palavras-Chaves, os termos "education data mining", "dropout" e "distance education" foram cadastrados no SR pelo professor. Em seguida, na etapa de cadastro de atividades, foi realizado o preenchimento de 3 questões/problemas e um simulado para a resolução dos alunos, conforme figura 6 .

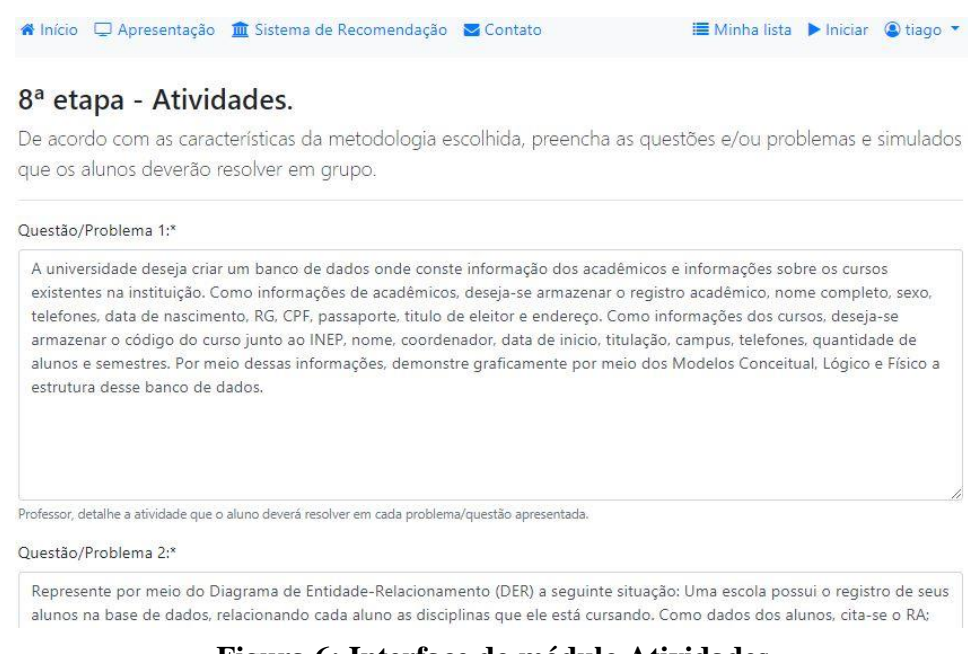

Figura 6: Interface do módulo Atividades

Para finalizar, a figura 7 apresenta o resultado da nona etapa, a exibição dos materiais complementares para a leitura ou OA obtidos através das palavras chaves cadastradas pelo professor e a Biblioteca BeautifulSoap. Nota-se que em cada material consta o nome da base de dados consultada, o título do trabalho e o link de acesso, bem como uma funcionalidade que permite o professor escolher entre exibir ou não o material retornado aos grupos de alunos formados.

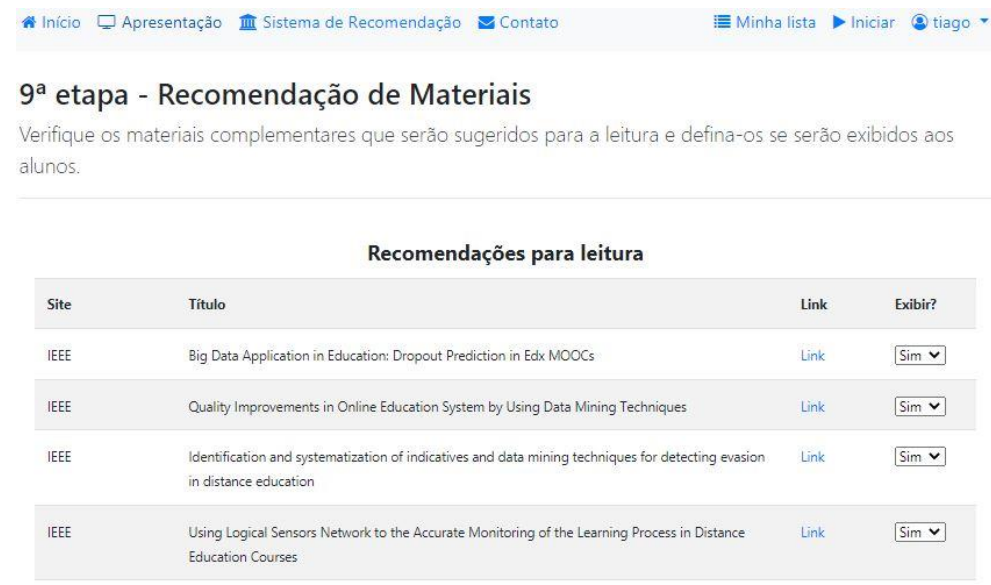

Figura 7: Interface do módulo Recomendação de Materiais

Portanto, além de indicação de materiais complementares, o SR propõe, como prática pedagógica, o uso de metodologias ativas com potencial de colaboração e troca de experiências e conhecimentos entre os estudantes.

A figura 8 apresenta a interface do aluno, em que são apresentados a ele os demais integrantes do grupo; link do Google Meet para a realização de reuniões on-line; atividades de testes simulados; materiais para leitura e ou utilização, como artigos e objetos de aprendizagens; e as atividades propostas pelo professor para a realização. 


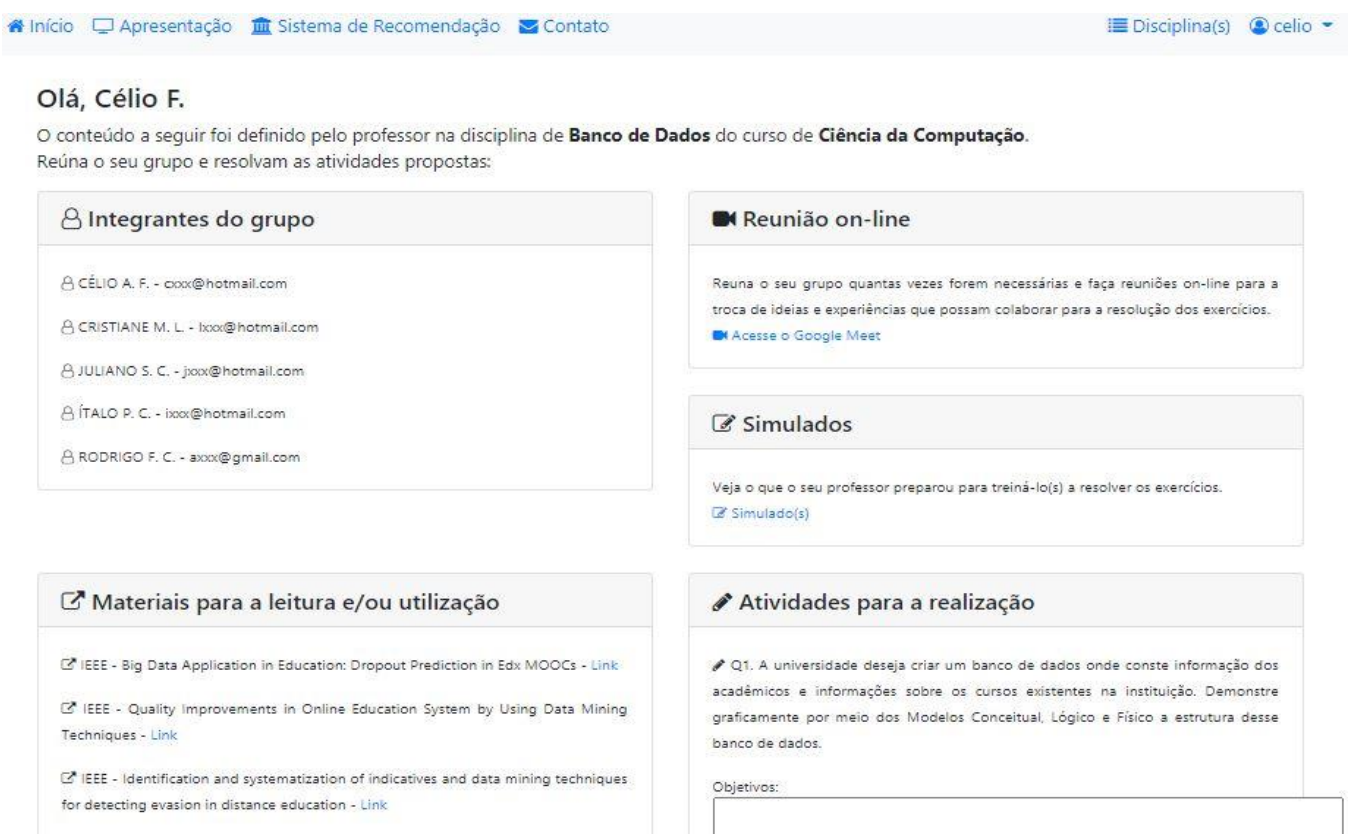

Figura 8: Interface do módulo do SR a nível de visão do aluno

\subsection{Avaliação de aceitação do SR}

Para a avaliação de aceitação foi realizado um estudo de caso com abordagem quantitativa. Foram disponibilizados um vídeo com exemplos de uso do sistema e um questionário contendo dez afirmações sobre o SR. Foram convidados professores de diferentes áreas de conhecimento de duas universidades. As respostas foram coletadas através da ferramenta Google Form. A avaliação utilizou como base os conceitos do modelo TAM, que avalia: a) Facilidade de uso percebida - grau em que uma pessoa acredita que o uso de um sistema de informação será livre de esforço; b) Utilidade percebida - grau em que uma pessoa acredita que o uso de um sistema pode melhorar o seu desempenho. As respostas foram padronizadas na escala Likert de cinco pontos, variando entre "Concordo fortemente", "Concordo", "Não concordo e nem discordo", "Discordo" e "Discordo fortemente". A tabela 1 apresenta as afirmações elaboradas de 1 a 5 sobre a facilidade de uso e 6 a 10 sobre à utilidade percebida.

Tabela 1: Questionário de avaliação

\begin{tabular}{|ll|}
\hline Item & Afirmação \\
\hline 1 & O SR é fácil de entender \\
2 & As informações da interface do SR são claras \\
3 & É possível usar com pouco esforço os recursos disponíveis no SR \\
4 & O SR reproduz a funcionalidade de recomendação de materiais \\
5 & A integração de serviços do SR proporciona uma maneira mais ágil e agradável de trabalhar \\
6 & O SR facilita a utilização de Metodologias Ativas \\
7 & O SR favorece a formação de grupos de alunos para a prática de ensino colaborativo \\
8 & O uso do SR facilita o trabalho do professor na tarefa de recomendar materiais complementares \\
9 & O uso do SR pode ajudar na mitigação dos riscos de evasão escolar \\
10 & Eu utilizaria o SR para o processo de ensino e aprendizagem \\
\hline
\end{tabular}

Participaram da pesquisa treze professores. Os resultados obtidos nas afirmações de 1 a 5 indicam que 49,2\% concordam fortemente, $38,5 \%$ concordam e 12,3\% não concordam e nem discordam sobre a facilidade de uso do SR, o que demonstra a satisfação de mais de $87 \%$ dos participantes. Não há respostas que discordam dessas afirmações. Em relação às afirmações de 6 a 10, que trata sobre a utilidade do SR, 38,5\% dos professores concordam fortemente, $38,5 \%$ concordam, 18,5\% não concordam e nem discordam, 3,1\% discordam e 1,5\% discordam fortemente. Os percentuais demonstram que $77 \%$ concordam que o SR pode ser útil para o 
processo de ensino e aprendizagem e de boa aceitação. A figura 9 apresenta os resultados das questões sobre facilidade de uso e utilidade percebida.
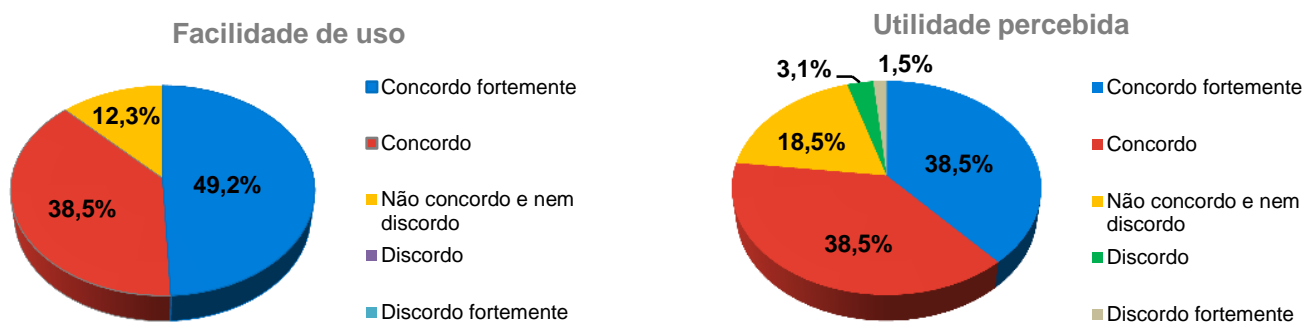

Figura 9. Resultados das questões sobre facilidade de uso e utilidade percebida.

Em relação à integração de Metodologias Ativas a um SR, os resultados da afirmação 6 demonstram o quanto o SR facilita a utilização de Métodos Ativos pelos professores, pois os percentuais de $38,5 \%$ foram observados nas respostas concordam e concordam fortemente, atingindo $77 \%$ a opinião do público participante da pesquisa.

$\mathrm{Na}$ questão 8 , os resultados indicam que 38,5\% dos professores concordam fortemente e que $53,8 \%$ concordam, o que mostra um alto grau de satisfação com o SR ao recomendar os materiais complementares para a leitura dos alunos, atingindo o objetivo do desenvolvimento do modelo e auxiliando no processo de aprendizagem.

Em relação à questão 9 , que relaciona a utilização do SR com a possibilidade de ajudar na mitigação dos riscos de evasão dos alunos, $46,2 \%$ dos professores responderam que não concordam e nem discordam, $38,5 \%$ concordam e 7,7\% concordam fortemente. Observa-se que o alto índice de não concordam e nem discordam pode significar a necessidade de tornar mais evidente à relação de identificação de alunos propensos a evadir, o uso de métodos ativos para o ensino colaborativo e a indicação de material complementar para a aprendizagem.

A figura 10 apresenta os resultados do questionário para as afirmações 6,8 e 9.
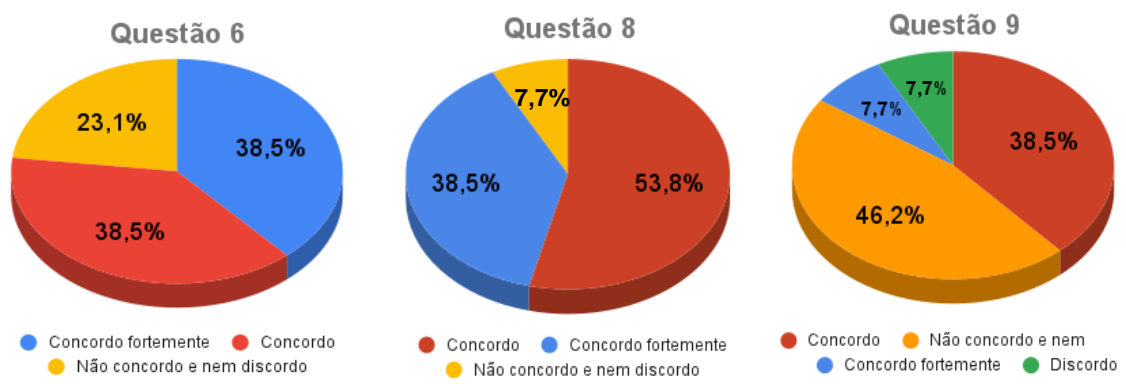

Figura 10. Resultados das questões 6, 8 e 9.

Com a finalidade de obter sugestões de melhorias, foi disponibilizado um espaço no questionário para o professor escrever livremente. Entre as sugestões, estão: a) formação de grupos: oportunizar a formação de grupos manualmente, por interesse, afinidade pessoal e/ou perfis psicológicos e emocionais; b) seleção de materiais: indicação manual de links.

\subsection{Análise crítica da proposta}

Conforme pesquisa realizada, nenhum dos trabalhos estudados considerou integrar a um SR as Metodologias Ativas após o uso das técnicas de MDE. É sabido que essas técnicas são amplamente utilizadas e tem contribuído significativamente no ensino. No entanto, o âmbito de maior contribuição tem sido a identificação de alunos propensos a evasão dos cursos. Nesse sentido, somados a essa ação, medidas de contenção desse elevado índice precisam ser tomadas. Como proposto, acredita-se que a utilização de Metodologia Ativa em um SR favorece a relação entre professores, tutores e alunos e torna o processo mais atraente e atualizado. Corroborando com a proposta aqui apresentada, trabalhos como Chandrasekaran et al. [2016], Leite e Ramos 
[2017], Lima e Siebra [2017], Leite et al. [2019] e Andrade et al. [2021] consideram a possibilidade de adicionar a Metodologia Ativa às técnicas para contribuir com a mitigação do abandono e o aumento da permanência dos alunos.

A Metodologia Ativa apoia os professores nas práticas de ensino e acompanhamento do processo de aprendizagem e desempenho das atividades desenvolvidas pelos alunos. Ao mesmo tempo, atua junto dos alunos estimulando-os a serem mais proativos e colaborativos em uma metodologia mais envolvente, participativa e envolvendo o contexto do grupo.

A avaliação realizada permitiu a verificação de funcionalidade e integração entre os componentes desenvolvidos, indicando a sua viabilidade como modelo de SR. Os resultados são promissores uma vez que o SR pode auxiliar o professor na seleção de materiais complementares e fomenta a relação entre os alunos para o processo de aprendizagem. Avaliações envolvendo alunos e professores em um contexto regular não foram realizadas tendo em vista a impossibilidade no período de pandemia ocorrido no ano de 2020 e 2021.

\section{Considerações finais}

Este trabalho apresentou um modelo de SR que utiliza Metodologias Ativas em alunos identificados através das técnicas de MDE como propensos a evadir e também mitigar essa possibilidade. Com o intuito de auxiliar o aluno e o professor no processo, várias funcionalidades são propostas, como a orientação de materiais de leitura complementar ao aluno e a possibilidade do professor escolher inicialmente entre Métodos Ativos a serem aplicados aos grupos formados pelo modelo de SR no processo de ensino e aprendizagem, de forma a inibir a possibilidade de evasão e aumentar o interesse pelo conteúdo e permanência do aluno.

Conforme estudos realizados, não foram encontradas evidências de SRs que integram Metodologia Ativa e MDE. Essa integração, avaliado no modelo de SR proposto, proporciona um avanço para esse processo, não apenas na identificação do aluno propenso a evadir do curso, mas também no apoio e melhoria da aprendizagem. Isso é fomentado pelo SR ao oportunizar a leitura de materiais complementares, a autonomia e experiências individuais, $\mathrm{o}$ compartilhamento de ideias em grupo, o desenvolvimento de habilidades sociais àqueles que têm dificuldade de aprendizagem centrada apenas no professor, a responsabilidade em aprender e a resolução de problemas. Com as metodologias ativas e técnicas inovadoras o professor consegue criar mecanismos para engajar e desafiar o aluno, gerando mais chances de fazer com que o estudante se sinta estimulado a permanecer no curso.

Diante disso, como trabalhos futuros, pretende-se a experimentação em larga escala do modelo proposto com as tecnologias destacadas, que envolvam diretamente alunos e professores de cursos diferentes. Também pretende-se acompanhar a vida acadêmica dos alunos identificados como propensos a evadir para verificar se o uso do SR com metodologia ativa foi capaz de incentivá-los a continuar os estudos. Por fim, melhorar as funcionalidades de formação de grupos e seleção de materiais complementares sugeridas pelos professores.

\section{Referências}

Acosta, O. C., Reategui, E. B., Behar, P. A. (2018). Recomendação de conteúdo em um ambiente colaborativo de Aprendizagem Baseada em Projetos. Revista Brasileira de Informática na Educação, 26: 91-111.

Almeida, C. M. M., Scheunemann, C. M. B., Santos, M. J., Lopes, P. T. C. (2019). Propostas de metodologias ativas utilizando Tecnologias Digitais e ferramentas metacognitivas para auxiliar no processo de ensino e aprendizagem. Revista Paradigma, 40: 204-220.

Alves, M. O., Medeiros, F. P. A., Melo, L. B. (2020). Levantamento do estado da arte sobre Aprendizagem baseada em Problemas na Educação a Distância e Híbrida. In: Simpósio Brasileiro de Informática na Educação (SBIE), p. 61-71.

Andrade, T. L., Rigo, S. J., Barbosa, J. L. V. (2021). Active Methodology, Educational Data Mining and Learning Analytics: A Systematic Mapping Study. Informatics in Education, 20(2): 171-204. 
Araujo, I. S.; Mazur, E. (2013). Instrução pelos colegas e ensino sob medida: uma proposta para o engajamento dos alunos no processo de ensino-aprendizagem de física. Caderno Brasileiro de Ensino de Física, 30(2): 362-384.

Bergmann, J., Sams, A. (2016). Sala de Aula Invertida: uma metodologia ativa de aprendizagem. LTC.

Breiman, L. (2001). Random Forests. Machine Learning, 45: 5-32.

Brito, M., Medeiros, F., Bezerra, E. P. (2019). An Infographics-based Tool for Monitoring Dropout Risk on Distance Learning in Higher Education. In: International Conference on Information Technology Based Higher Education and Training (ITHET). IEEE.

Campos, A., Hollerweger, L., Santos, G., Farias, A. F., Behar, P. A. (2017). Mapeamento de soluções tecnológicas em sistemas de recomendação educacionais em âmbito brasileiro. Informática na Educação: teoria e prática, 20(3): 79-96.

Chandrasekaran, D., Thirunavukkarasu, G. S., Littlefair , G. (2016). Collaborative Learning Experience of Students in Distance Education. In: International Symposium on Project Approaches in Engineering Education and Active Learning in Engineering Education Workshop. p. 90-99.

Cunha, F. O. M., Siebra, C. A. (2016). Mapeamento sistemático na literatura acadêmico-científica sobre abordagens para a formação de grupos em E-Learning. Revista Brasileira de Informática na Educação, 24(3): 17-30.

Ferreira, L. G. A., Barbosa, J. L. V., Gluz, J. C., Vicari, R. (2015). UbiGroup: um modelo de recomendação ubíqua de conteúdo para grupos dinâmicos de aprendizes. Revista Brasileira de Informática na Educação, 23(3): 40-55.

Guo, R., Li, L., Han, M. (2018). On-demand virtual lectures: Promoting active learning in distance learning. In: International Conference on E-Education, E-Business and E-Technology, p. 1-5. ACM.

Kostopoulos, G., Karlos, S., Kotsiantis, S. (2019). Multiview Learning for Early Prognosis of Academic Performance: A Case Study. IEEE Transactions on Learning Technologies, 12(2): 212-224. IEEE.

Leite, L. S., Ramos, M. B. (2017). A metodologia ativa no Ambiente Virtual de Aprendizagem. Metodologia ativa na educação, 85-101. Pimenta Cultural.

Leite, R. R., Pitangui, C. G., De Assis, L. P., Andrade, A. V. (2019). Sistemas de Recomendação em Ambientes Educacionais: estado da arte e perspectivas futuras. In: Simpósio Brasileiro de Informática na Educação (SBIE), p. 109-118.

Lewis, D. D. (1998). Naive Bayes at forty: The independence assumption in information retrieval. In: Machine Learning: European Conference on Machine Learning (ECML), p 4-15.

Lima, E., Siebra, C. (2017). CollabEduc: Uma Ferramenta de Colaboração em Pequenos Grupos para Plataformas de Aprendizagem a Distância. In: Simpósio Brasileiro de Informática na Educação (SBIE), p. 1707-1716.

Lima, J. V. V., Silva, C., Alencar, F., Santos, W. (2020). Metodologias Ativas como forma de reduzir os desafios do ensino em Engenharia de Software: diagnóstico de um survey. In: Simpósio Brasileiro de Informática na Educação (SBIE), p. 172-181.

Marangunić, N., Granić, A. (2014). Technology acceptance model: a literature review from 1986 to 2013. Universal Access in the Information Society, 14: 81-95. Springer.

Marques, L. T., Castro, A. F., Marques, B. T., Silva, J. C. P., Queiroz, P. G. G. (2019). Mineração de dados auxiliando na descoberta das causas da evasão escolar: um mapeamento sistemático da literatura. Revista Novas Tecnologias na Educação, 17(3): 194-203.

Pallof, R. M., Pratt, K. (2002). Estimulando a Aprendizagem Colaborativa. In: Construindo Comunidades de Aprendizagem no Ciberespaço: estratégias eficientes para salas de aula on-line. Porto Alegre: Artmed.

Queiroga, E. M., Cechinel, C., Aguiar, M. S. (2019). Uma abordagem para predição de estudantes em risco utilizando algoritmos genéticos e mineração de dados: um estudo de caso com dados de um curso técnico a distância. In: Simpósio Brasileiro de Informática na Educação (SBIE), p. 119-128.

Ramos, J. L. C., Silva, J. C. S., Prado, L. C., Gomes, A. S., Souza, F. F. D., Zambom, E. G., Rodrigues, R. L. (2017). Um Modelo Preditivo da Evasão dos Alunos na EAD a partir dos Construtos da Teoria da Distância Transacional. In: Simpósio Brasileiro de Informática na Educação (SBIE), p. 1227-1236. 
Ramos, J. L. C., Silva, J. C. S., Prado, L. C., Gomes, A. S., Rodrigues, R. L. (2018). Um estudo comparativo de classificadores na previsão da evasão de alunos em EAD. In: Simpósio Brasileiro de Informática na Educação (SBIE), p. 1463-1472.

Rolim, V. B., Mello, R. F. L., Costa, E. B. (2017). Utilização de técnicas de aprendizado de máquina para acompanhamento de fóruns educacionais. Revista Brasileira de Informática na Educação, 25(3): 112130.

Santos, R. M. M., Pitangui, C. G., Andrade, A. V., Assis, L. P. (2016). Uso de Séries Temporais e Seleção de Atributos em Mineração de Dados Educacionais para Previsão de Desempenho Acadêmico. In: Simpósio Brasileiro de Informática na Educação (SBIE), p. 1146-1155.

Silva, J. T., Silva, I. M. (2020). Uma revisão sistemática sobre a aprendizagem baseada em problemas no ensino de Ciências. Pesquisa e Ensino, 1: 1-29.

Waheed, H., Hassan, S., Aljohani, N. R., Hardman, J., Alelyani, S., Nawaz, R. (2020). Predicting academic performance of students from VLE big data using deep learning models. Computers in Human Behavior, 104: 1-13. Elsevier.

Widyahastuti, F., Tjhin, U. (2018). Performance Prediction in Online Discussion Forum: state-of-the-art and comparative analysis. In: International Conference on Computer Science and Computational Intelligence. p. 302-314. Elsevier. 